\title{
Energetic photons applied to COVID-19 Coronavirus S2 subunit of spike glycoprotein for host cell entry inhabitation: An alternative approach for conventional vaccination
}

\section{Z. Y. Khattari ( $\square$ zkhattari@hu.edu.jo )}

Hashemite University

\section{Abu Al-Sayed \\ Isra University \\ F. Afaneh}

Hashemite University

M. Maghrabi

Hashemite University

S. Al-Omari

Hashemite University

J. Al-Jundi

Hashemite University

\section{Research Article}

Keywords: Covid-19, SARS-CoV-2, Spike protein, vaccination, cell, resonance energy, buildup factors.

Posted Date: March 24th, 2021

DOl: https://doi.org/10.21203/rs.3.rs-232923/v1

License: (c) (1) This work is licensed under a Creative Commons Attribution 4.0 International License.

Read Full License 


\section{Abstract}

We have investigated numerically the ability to inhibit spike protein from SARS-CoV-2 to attach and inter the host cell when exposed to energetic photons. The Geometric Progression fitting method have been adopted to calculate the equivalent atomic number for photon energy absorption $\left(\mathrm{Z}_{\text {eq }}\right)$, exposure and absorption buildup factors in the energy range $E \in[15-300] \mathrm{keV}$ for the S2-subunit in the spike protein. The buildup factors have shown a peak value at adsorption resonance energy between 36-60 eV per amino acid of the S2-subunit which depends on the mean free path of the photon within the protein structure and the type of mutation. The resonance energies (between UV and X-ray range) have been found to depend on the protein molecular composition. This opens the possibility of using energetic photons to break up the S2-subunit into small fragments.

Our results may contribute to the continues racing for finding noninvasive technique for medical trials using radiotherapy treatment for the COVID-19 virus.

\section{Introduction}

During the last two decades several variants of coronaviruses have caused serious health threats to humanity[1-7]. Among them, severe acute respiratory syndrome coronavirus (SARS-CoV), in 2003 [1-2], Middle East respiratory syndrome coronavirus (MERS-CoV) in 2012 [3], porcine epidemic diarrhea coronavirus in 2013 [4], and finally the 2019novelcoronavirus, COVID-19, (officially known as SARS-CoV2). COVID-19 is a newly emerged human infectious coronavirus that was originated in Wuhan seafood market and quickly spread beyond China [5-6]. In general, coronaviruses cause widespread respiratory and central nervous system diseases in humans [7-9]. Coronaviruses can adapt to new environments through mutation and recombination with relative ease and hence are programmed to alter host range and tissue tropism efficiently [10-11]. The World Health Organization (WHO) has declared COVID-19as a pandemic in March 2020. Up to date over 105 million cases have been reported worldwide, and more than two million casualties. Very recently the U.S. Food and Drug Administration (FDA) has approved two antiviral vaccines against SARS-CoV-2. The first one is due to the Pfizer and BioNTech, while the other one is due to Moderna. Both vaccines have shown a $95 \%$ efficiency against this pandemic virus [23].

Although many resent statistical surveys and clinical tests are being rushing on COVID-19 across the globe $[12,13]$, yet no clue from the available clinical controlled

tests has proven that it may enhances the therapy conclusions about the patients end results [14]. As the pandemic escalate $s$, it is an essential issue to discover a exceptional therapeutic for COVID-19, and vaccines pledge to different variants of SARS-CoV-2 proteins. This protein types are a family of a monostranded enveloped positive-sense RNA virus [15]. The entire RNA sequencing genome procedure revealed that such stranded-RNA is recognized by 29,881 bp while encoding 9860 amino acids (GeneBank no. MN908947) [16]. Moreover, these specific coronavirus proteins via gene fragmentation method uncovers 
there structural and nonstructural secondary conformations. In particular, SARS-CoV-2 virus encompasses four distinct

structural proteins: Spike, Nucleocapsid, Envelope and Membrane protein [17]. Amongst them, the Spike (S-) glycoprotein is a giant, transmembrane and multifunctional protein. This S-protein compete with other coronavirus proteins by mediating itself in an deadly and vital role with respect to host cell via viral attachment, fusion and entry into it $[17,18]$. The S-protein has two sub-domains namely, S1 and S2 [19, 20], which are then fissure into various biologically active subunits. Of interest, the S1-subunit which runs the binding to the host cell receptor which finally fascinating the cell entry $[19,20]$. On the other hand, the whole virus initiation and fusion entry into the host cellular membrane is governed by the S2 portion of the spike protein. During this process the viral contains of the virus is being injected into the infected cell $[21,22]$. Furthermore, It has been recently concluded that the coronaviruses adapted the circumvention method to trick the host immune system of the infected host cells using conformational masking and glycan shielding of its own S-protein[22].

Several physical and chemical techniques $[28,29]$ have been applied to inactivate the virus. Such techniques include heating, UV light and ionizing radiation methods. Each rout has its own drawback regarding epitopes damage and development during medical treatment. Gamma irradiation might be considered as a very safe and effective method as indicated in many recent research articles (for full review see [28] and the references therein). On the other hand, the electromagnetic (EM) spectrum between the UV (i.e., $10^{18} \mathrm{~Hz}$ ) and the X-ray radiation (i.e., $10^{19} \mathrm{~Hz}$ ) is very sensitive to molecular vibration between groups of atoms in macromolecules [30]. Since the resonance absorption energy depends on the protein backbone molecular structure of the amino acids, then each molecule produces its own vibration resonance frequency signature which may lead to the breaking up of the protein structure at specific position along its backbone [31, 32].

Many researchers have employed the five-parameter geometric progression (G-P) fitting method which is originally developed and pioneered by Berger and Hubbell [24] to determine both EABF and EBF for amino acids, single-chain fatty acids and carbohydrates and other non-biological compounds [25]. These studies have shown that G-P fitting is a convenient method for estimating the energy absorption and exposure buildup factors (i.e., EABF and EBF) for many biological molecules. These two important factors may be used to pinpoint the resonance frequency or energy of the viral RNA or DNA strand. Scanning the energy range from few keV up to hundreds of keV may enable the determination of the breaking-up energy of specific sub-domains of the protein in the target.

Large volume of publications has appeared in literature as a consequence of the development of WinXCom software. Manohara and Hanagodimth [26] in couples of independent research reports have determined the effective atomic numbers and electron densities of some essential amino acids in a broad range of photon energies from a few keVup to $100 \mathrm{GeV}$. A progressive investigation has also been performed by Kurudireket al. [27] who investigated the effect of y-ray energy on some important human tissues by calculating the absorption and exposure buildup factors. On further attempt of the same 
research group, Kurudirek and Onara [28] have detailed the $y$-ray energy absorption (EABF) and exposure buildup factors (EBF) for different biological-related molecules in the energy range $E \in$ [15-1500] $\mathrm{keV}$ up to a $40 \mathrm{mfp}$. Most of these "in vitro" experiments have been performed to understand what is happing at sub-cellular level when $\mathrm{X}$ - or $\mathrm{Y}$-ray interacts with proteins, tissues, or cell membrane [29].

In the present work, we are motivated by the current research work onCOVID-19 vaccination development around the globe using the conventional clinical treatment. The roles of the SARS-CoV-2 spike glycoprotein in receptor binding and membrane fusion make it an ideal target for vaccine and antiviral development. The development of SARS vaccines based on the spike protein has been summarized in [33-37]. Here, we propose a novel approach to inhabit the S-protein from entering the host cell by applying UV and X-ray irradiation. Thereby, we have investigated theoretically the effect of photon irradiation on the amino acids building blocks in the SARS-CoV-2 spike protein (see Schematic 1) for possible inhabitation of virus to enter and relocate itself into the host cell. This approach is based on the resonance energy for S2-subdomain of the spike protein to break it up into small fragments (see schematic 1) [18-20]. The molecular formula of the nucleotide bases of S2 protein from different coronaviruses mutations used in this study are summarized in Table 1.

\section{Theoretical And Computational Background}

The buildup factor values and the G-P fitting parameters of the S2-subdomian were calculated using the well-known method of logarithmic interpolation from the equivalent atomic number $\left(Z_{\text {eq }}\right)[37,38]$. The computational work has been conducted in three steps. The first step concerns with the computation of the $Z_{\text {eq }}$ values for S2-subdomain. The second step deals with the calculations of G-P fitting parameters and finally the computation of the buildup factor values are illustrated in step three.

\subsection{Calculation of the equivalent atomic number $Z_{e q}$}

The $Z_{\text {eq }}$ of a particular material is defined as the ratio $R(\mu / \rho)_{\text {Compton }} /(\mu / \rho)$ Total of that material at a specific photon energy corresponding to the ratio of an element at the same photon energy. Thus, firstly the Compton partial mass attenuation coefficients, $(\mu / \rho)_{\text {Compton }}$ and the total partial mass attenuation coefficients $(\mu / \rho)_{\text {Total }}$ were obtained for elements of atomic number Zî [4- 40]. These two ratios were also calculated for the five nucleotide bases for the incident photon energy $E \hat{l}[15-300] \mathrm{keV}$ using XCOM; Photon Cross Sections Database, Web Version 3.1 program $[38,39]$. The logarithmic interpolation of $Z_{\text {eq }}$ has been performed using the relation $[40,41]$

$$
Z_{e q}=\frac{Z_{1}\left(\log R_{2}-\log R\right)+Z_{2}\left(\log R-\log R_{1}\right)}{\log R_{2}-\log R_{1}}
$$


where $Z_{1}$ and $Z_{2}$ are the atomic numbers of the elements corresponding to the ratios $R_{1}$ and $R_{2}$ respectively. The ratio $R$ for a nucleotide base satisfies the following inequality $R_{2}<R<R_{1}$ at a specific photon energy.

\subsection{Calculation of the geometric progression (G-P) fitting parameters}

In the second step we used the standard reference database recently released by the American National Standards ANSI/ANS-3.1 [22] which provides the various buildup factor (or consequently G-P fitting parameters) for 23 chemical elements, one compound (i.e., $\mathrm{H}_{2} \mathrm{O}$ ) and two mixtures (i.e., air and concrete) in the energy range of $E \hat{I}[15-300] \mathrm{keV}$. This database has covered the G-P fitting parameters for penetration depths up to $40 \mathrm{mfp}$. Then, the G-P fitting buildup factor coefficients of the five nucleotide bases used in this study were then interpolated according to the following formula [22]:

$$
P=\frac{P_{1}\left(\log Z_{2}-\log z_{e q}\right)+P_{2}\left(\log z_{e q}-\log z_{1}\right)}{\log Z_{2}-\log Z_{1}}
$$

where $P_{1}$ and $P_{2}$ are the values of the G-P fitting coefficients corresponding to elements of atomic numbers $Z_{1}$ and $Z_{2}$ respectively at a given photon energy; $Z_{\text {eq }}$ is the equivalent atomic number of S2subdomain. The effective atomic number $\left(Z_{e f f}\right)$ is related to $s_{a}$ and $s_{e}$ through the following relation [22, 23]

$$
Z_{\text {eff }}=\frac{\sigma_{a}}{\sigma_{\varepsilon}}
$$

where $s_{a}$ is the effective atomic cross section and $s_{e}$ is the total electronic cross section [22].

\subsection{Calculation of energy and exposure buildup factors}

Finally, the computed G-P fitting parameters $\left\{\mathrm{P}_{1}, \mathrm{P}_{2}\right\}$ were then used to generate the energy buildup factors (i.e., absorption and exposure) for the five nucleotide bases at selected incident photon energies in the range $15<E<300 \mathrm{keV}$ up to $40 \mathrm{mfp}$ penetration depths. This was performed using the G-P fitting formula according to the following piecewise function [22,23]

$$
B(E, x)=\left\{\begin{array}{cc}
1+(b-1) x, & K=1 \\
1+\frac{b-1}{K-1}\left(K^{x}-1\right), & 1<K \leq 40 \\
\text { otherwise, } & K>40
\end{array}\right.
$$


The function $K(E, x)$ which represents the photon dose multiplication factor is rather a complicated tangent hyperbolic function and is given by:

$$
K(E, x)=c x^{a}+d \frac{\tanh \left(x / x_{k}-2\right)-\tanh (-2)}{1-\tanh (-2)}, x \leq 40
$$

where $E$ is the incident photon energy and $x$ is the penetration depth in units of $\operatorname{mfp}$. The letters $\{a, b, c, d$, $\left.x_{k}\right\}$ are the G-P fitting parameters. The parameter $b$ represents the value of the buildup factor at $1 \mathrm{mfp}$. These parameters are usually obtained by the least-square fitting procedure. The dose function $K(E, x)$ strongly depends on the incident photon energy $E$ and the penetration depth $x$.

\section{Results And Discussion}

The molecular formulae of SARS-CoV-2 spike glycoprotein mutations spanning the S2-subdomain investigated in this study are presented in Table 2. The composition of each mutation will be correlated with the EBF and EABF in the subsequent discussion (see below). In addition, the change in the effective atomic number $\left(\mathrm{Z}_{\mathrm{eq}}\right)$ as a function of the incident photon energy for each mutation is shown in Table 3 and the supplementary materials provided with this report. Moreover, the change in the equivalent atomic number with incident photon energy $\left(Z_{e q}\right)$ for S2-subdomain along with the G-P fitting parameters for the various mutations in the energy range $\hat{E}[15-300] \mathrm{keV}$ are shown.

It is clear from Table 3 that the only atomic weight fraction between the S2-subdomain constituents that may satisfy this proportion is the $\mathrm{H}$-atom number. Therefore, we infer that the variation of $\mathrm{Z}_{\text {eq }}$ between the S2-subdomains for the various mutations can be correlated with the number of hydrogen atoms presented in each compound. Meanwhile, other atomic weight fractions cannot satisfy the observed behavior of $Z_{\text {eq }}$ of the $S 2$-subdomain for all mutations used in this study. The energy exposure (EBF) and absorption (EABF) buildup factors for S2-subdomain are shown in Fig. 1 and Fig. 2 as a function of the incident photon energy for mean free path $(m f p)$ values $m f p$ i $[1,40]$. Information extracted from these two figures are then used to compare the effect on S2-subdomain in different mutations as a function of the incident photon energy at specific mfp values.

\section{Radiation buildup factors of the S2-subdomain}

Figure 1a-1e gives a comprehensive picture about the EBF for S2-subdomain of different SARS-CoV-2 mutations studied here as a function of energy at specific mean free path $(m f p)$ values as indicated in the Figure. The general conclusions drawn from this figure can be summarized as 1 ) the calculated values of EBF start to decline with increasing the $m f p$ value nearly in the same energy range regardless of the mutation used in this study, 2) the Figure also shows that EBF globally having a Gaussian-like distribution with skews to the left indicating the domination of the lower than higher photon energy when living matter interacts with radiation. and 3 ) the distribution peak shifts to the right as the $m f p$ is increased where one of the three well-known scattering processes would dominate the others (will be 
commented on later). The energy peak shift might be correlated with the resonance energy or frequency of the S2-subunite that may lead to breaking up the bonds along the backbone of this part of the spike protein. This also may involve the different bonding energies between all the S2-subunite molecular constitutes such as Carbon, Oxygen, Hydrogen and Nitrogen that might be the exact resonance energy between any of these atoms. For low mpf values, the resonance energy is estimated to be about $60 \mathrm{keV}$ per S2 subunit (equivalent to $36 \mathrm{eV}$ per amino acid residue) [28]. This energy corresponds to $9^{\prime} 10^{18} \mathrm{~Hz}$ (or $\mathrm{I}=0.344 \AA$ ) which lies between the UV and X-ray region in the electromagnetic radiation spectrum. For the other $m p f$ values, this resonance frequency depends on the value of mpf. Many theoretical predictions of the resonance absorption spectra of biological macromolecules are in the UV-X-ray range [28-30]. Thus the EM waves offer multiple resonance frequencies that may excite the low-frequency part of the internal molecular structure of the S2-protein. These internal vibrations include atoms within the amino acid molecules linked via weak bonds (e.g. van der Walls and hydrogen bonds) $[41,42]$.

The variation of energy absorption exposure buildup (EABF) factor versus the incident photon energy at selected penetration depths for all S2-subdomain is depicted in Fig. 2a-2e. Obviously, all S2-subdomain consistently show the same general trend of variations of EABF with the incident photon energy. The variations in the buildup factors as a function of the incident photon energy show three distinct regions depending on the interaction process between the incident photon with matter.

The buildup factors show a rapid increase in the low energy region, reaching a maximum then decrease at a much lower rate when the incident photon energy is increased further. It is worth noting that the buildup factors for S2-subdomain coincide in the low energy region and deviate at higher energy regardless of the mutation or the $m f p$ values. The observed variations can be easily explained by adopting the three well-known processes of photon interaction with matter. The photoelectric effect is the dominant process at the low energy side of the spectrum and operates for a short period of time resulting in small buildup factor values. On the other hand, Compton scattering is the dominant process in the energy region (100-300 keV) and lasts for longer time. The probability of Compton scattering decreases as the photon energy increases. In the intermediate region around the peak maximum the two processes compete with each other. Compton scattering can maximally enhance both buildup factors by multiple photon scattering inside the S2-subdomain intermolecular structure. For example, the largest EBF and EABF values occur around $60 \mathrm{keV}$ (or $36 \mathrm{eV}$ per amino acid) at $1 \mathrm{mfp}$, and around $100 \mathrm{keV}$ (or $60 \mathrm{eV}$ per amino acid) at $40 \mathrm{mfp}$ [28]. The third process in which the incident photon interacts with matter is the pair production. This process was excluded as the maximum photon energy used in this study is $300 \mathrm{keV}$ which is well below the threshold energy $(1.02 \mathrm{MeV})$ for pair production to occur. The energy of peak maximum (peak of the buildup factors vs energy) may be considered as a resonance energy that the macromolecule may adsorb before it breaks up into small fragments (see the discussion below).

Our results show that the buildup factors depend on the chemical composition of S2-subdomain for the entire energy region studied here. In addition, it is also observed that the buildup factors attain their largest values for penetration depth of $40 \mathrm{mfp}$, which is the limit of our calculations. In fact, for $m f p>40$ another mathematical and theoretical formalism must be adopted to gain insight about their variations 
with energy or $m f p$ [22]. The equivalent atomic number $Z_{\text {eq }}$ takes the values between 6.04 and 4.44 for all mutations and steadily decrease with the incident photon energy.

The above results can be discussed in view of the vibrational adsorption frequencies exhibited within the molecular structure of the S2-subunite [28, 41,42$]$. It is know that the function and conformational kinetics of proteins and peptides are governed by the intermolecular forces hidden in the hydrogen bonding like amide and carbonyl $\mathrm{C}=0$ groups and other functional groups present in the protein backbone. The presence of multiple hydrogen bonds in the secondary structure of the protein under study results in stabilizing and strengthen the molecular strands of the $\mathrm{S} 2$ subunit. This in part may explain the anticipated higher binding energy calculated in this report which came collective from all hydrogen and non-hydrogen bonds present within the protein structure. These bonds together determine the expected 3D-molecluer structure, vibrational resonance frequencies along with the conformational changes in the S2-subunit when exposed to a unique environment that affect its molecular structure [40, 41]. Obviously, each protein or peptide possesses a unique resonance absorption energy that can be used to identify the breaking frequency of the various mutations in the S-protein based on such spectroscopic theoretical calculations. Our data clearly demonstrate that the molecular structure of the studied S2-subdomain is a crucial factor for possible radiation treatment of COVID-19 using energetic photons that lie in the UV-X-ray region of the EM spectrum. The data presented here for exposure and adsorption buildup factors for the S-protein opens the possibility to break up this molecule into small fragments by using photons with specific energy $[42,43]$.

Along these lines of finding and of particular interest is the treatment of COVID-19 with X-ray therapy which dated back to 1939 [43-45]. The recent studies using modern technology recommended a single dose of 0.5-1.0 Gy and a whole doses of 3.0-6.0 Gy in 2 or 3 fractions / week [44]. In fact, high X-ray radiation doses may initiate or lead to DNA and RND damage resulting in a cell death. Since the majority of death cases of COVID-19 is due to a failure in the cardiopulmonary dysfunction, it becomes necessary to recommend low-dose radiotherapy to patients with COVID-19 as it reduces endothelial-leukocyte interaction. This process may activate T-cells and change the polarization of macrophage in response to X-ray treatment [45]. Feldman et. al. [Am J. 2019, 46] have applied gamma irradiation for a possible inactivation of the emergent vial pathogen namely COVID-19. They used ${ }^{60} \mathrm{Co}$ source to inactivate infectious diseases by destructing multiple replicates of the nucleic acid either directly or by radiocleavage of the genetic materials of SARS-Cov-2 proteins. They have tested the inactivation of selected viral pathogens in liquid medium containing T-proteins from different members of virus families and infectious agents in different mixtures. Their conclusion require a defined peak of the absorbed gamma ray doses to fully inactivate these enveloped protein within these viruses. They also found that this peak depends on different factors such as virus susceptibility to gamma irradiation, genome size and virus type and composition [46].

\section{Conclusions}


Valuable information have been gathered about the equivalent atomic number of S2-subdomain at different energies and $m f p$. Moreover, the photon buildup factors (i.e., EBF and EABF) have been numerically calculated for this life-essential molecules in the energy range $\hat{\theta}[15-300] \mathrm{keV}$ up to ultimate penetration depths of $40 \mathrm{mfp}$. For medical trails applications, we observed, within the energy range studied in this report, the existence of different energy resonance frequencies for the S2-subdomain in various mutations. This adsorption resonance energy may be used to break up this type of S-proteins and prevents it entry to the host cell. The calculated resonance frequencies depend on the molecular structure, the environmental conditions and the type of mutation in the Spike protein. The calculated resonance energy values are in the range of 36-60 eV per amino acid. The current study showed that the presence of the S2-subdomain within the structure of SARS-CoV-2 virus plays a vital role in their response to external stimuli and breaking the S2-subunit into small fragments when exposed radiation (see Schematic 1).

\section{Declarations}

\section{Acknowledgment}

The financial support from the Hashemite University is gratefully acknowledged.

\section{Author Contributions}

Z.KH.: Conceptualization, Writing the original draft, preparation and Methodology. M.A. \& J. A.: Data curation, numerical analysis. S.A. \& F.A: Validation, Visualization, and reproducing the figures and tables. M. M. Writing- Reviewing and finalizing the manuscript.

\section{Conflict of Interests Statement}

The authors declare that there is no conflict of interest relevant to the current manuscript.

\section{References}

1. Ksiazek TG, Erdman D, Goldsmith CS, Zaki SR, Peret T, et al. 2003. A novel coronavirus associated with severe acute respiratory syndrome. N. Engl. J. Med. 348:1953-66

2. Rota PA, Oberste MS, Monroe SS, Nix WA, Campagnoli R, et al. 2003. Characterization of a novel coronavirus associated with severe acute respiratory syndrome. Science 300:1394-99

3. de Groot RJ, Baker SC, Baric RS, Brown CS, Drosten C, et al. 2013. Middle East respiratory syndrome coronavirus (MERS-CoV): announcement of the Coronavirus Study Group. J. Virol. 87:7790-92

4. Chen Q, Li G, Stasko J, Thomas JT, StenslandWR, et al. 2014. Isolation and characterization of porcine epidemic diarrhea viruses associated with the 2013 disease outbreak among swine in the United States. J. Clin. Microbiol. 52:234-43

5. Zhu N, Zhang D, Wang W, Li X, Yang B, Song J, et al. A novel coronavirus from patients with pneumonia in China, 2019. N Engl J Med. 2020;382:727-33. 
6. Wu C, Liu Y, Yang Y, Zhang P, Zhong W, Wang Y, et al. Analysis of therapeutic targets for SARS-CoV-2 and discovery of potential drugs by computational methods. Acta Pharm Sin B. 2020;10:766-88.

7. Enjuanes L, Almazan F, Sola I, Zuniga S. 2006. Biochemical aspects of coronavirus replication and virus-host interaction. Annu. Rev. Microbiol. 60:211-30

8. Perlman S, Netland J. 2009. Coronaviruses post-SARS: update on replication and pathogenesis. Nat. Rev. Microbiol. 7:439-50

9. Aiping Wu et. al. 2020.Genome Composition and Divergence of the Novel Coronavirus (2019-nCoV) Originating in China. Cell Host \& Microbe. 27:325-328

10. Graham RL, Baric RS. 2010. Recombination, reservoirs, and the modular spike: mechanisms of coronavirus cross-species transmission. J. Virol. 84:3134-46

11. Li WH,Wong SK, Li F, Kuhn JH, Huang IC, et al. 2006. Animal origins of the severe acute respiratory syndrome coronavirus: insight from ACE2-S-protein interactions. J. Virol. 80:4211-19

12. Kirchdoerfer RN, Cottrell CA, Wang N, Pallesen J, Yassine HM, et al. 2016. Pre-fusion structure of a human coronavirus spike protein. Nature 531:118-21

13. Wu C, Liu Y, Yang Y, Zhang P, Zhong W, Wang Y, et al. Analysis of therapeutic targets for SARS-CoV-2 and discovery of potential drugs by computational methods. Acta Pharm Sin B. 2020;10:766-88.

14. Liu C, Zhou Q, Li Y, Garner LV, Watkins SP, Carter LJ, et al. Research and development on therapeutic agents and vaccines for COVID-19 and related human coronavirus diseases. ACS Cent Sci. 2020;6:315-31.

15. Sanders JM, Monogue ML, Jodlowski TZ, Cutrell JB. Pharmacologic treatments for coronavirus disease 2019 (COVID-19): a review. JAMA. 2020;323:1824-36.

16. Lu R, Zhao X, Li J, Niu P, Yang B, Wu H, et al. Genomic characterization and epidemiology of 2019 novel coronavirus: implications for virus origins and receptor binding. Lancet. 2020;395:565-74.

17. Chen L, Liu W, Zhang Q, Xu K, Ye G, Wu W, et al. RNA based mNGS approach identifies a novel human coronavirus from two individual pneumonia cases in 2019 Wuhan outbreak. Emerg Microbes Infect. 2020;9:313-9.

18. Vincent, M. J., Bergeron, E., Benjannet, S., Erickson, B. R., Rollin, P. E., Ksiazek, T. G., Seidah, N. G., \& Nichol, S. T. (2005). Chloroquine is a potent inhibitor of SARS coronavirus infection and spread. Virology Journal, 2(1), 69-10. https://doi.org/10.1186/1743-422X-2-69

19. Walls, A. C., Park, Y.-J., Tortorici, M. A., Wall, A., McGuire, A. T., \& Veesler, D. (2020). Structure, function, and antigenicity of the SARS-CoV-2 Spike glycoprotein. Cell, 181(2), 281-212.

20. Wang, M., Cao, R., Zhang, L., Yang, X., Liu, J., Xu, M., Shi, Z., Hu, Z., Zhong, W., \& Xiao, G. (2020). Remdesivir and chloroquine effectively inhibit the recently emerged novel coronavirus (2019-nCoV) in vitro. Cell Research, 30(3), 269-271.

21. Wrapp, D., Wang, N., Corbett, K. S., Goldsmith, J. A., Hsieh, C.-L., Abiona, O., Graham, B. S., \& McLellan, J. S. (2020). Cryo-EM structure of the 2019-nCoV spike in the prefusion conformation. Science, 367(6483), 1260-1263. 
22. Tian, X., Li, C., Huang, A., Xia, S., Lu, S., Shi, Z., Lu, L., Jiang, S., Yang, Z., Wu, Y., \& Ying, T. (2020). Potent binding of 2019 novel coronavirus spike protein by a SARS coronavirus-specific human monoclonal antibody. Emerging Microbes \& Infections, 9(1), 382-385.

23. Anshumali Mitta, Kavyashree Manjunath, Rajesh Kumar Ranjan, Sandeep Kaushik, Sujeet Kumar, Vikash Verma (2020). COVID-19 pandemic: Insights into structure, function, and hACE2 receptor recognition by SARS-CoV-2. PLOS Pathogens 1,1-19

24. WHO Director-General 's remarks at the media briefing on 2019-nCoV on 11 February (2020). (n.d.). Retrieved from https://www.who.int/dg/ speeches/detail/who-director-general-s-remarks-at-themedia-briefingon- 2019-ncov-on-11-february-2020

25. Berger MJ, Hubbell JH, Seltzer SM, Chang J, Coursey JS, Sukumar R, Zucker DS, Olsen K. XCOM: Photon Cross Sections Database, Web Version 3.1, National Institute of Standards and Technology, Gaithersburg, MD, USA, November 2010, Available from: <http://www.nist.gov/pml/data/xcom/>, (Originally published as NBSIR 87-3597, XCOM: Photon Cross Sections on a Personal Computer, July 1987).

26. Manohara SR, Hanagodimath SM. 2007 Studies on effective atomic numbers and electron densities of essential amino acids in the energy range $1 \mathrm{keV}-100 \mathrm{GeV}$ Ncul. Instrum. Methods Phys. Res. B 258:321-28.

27. Kurudirek M, Dogan B, Ingec M, Ekinci N, Ozdemir Y. 2011. Gamma-ray energy absorption and exposure buildup factor studies in some human tissues with endometriosis. Appl. Radiat. Isot 69:381-388.

28. Kurudirek M, Yuksel Ö. 2011. A comprehensive study on energy absorption and exposure buildup factors for some essential amino acids, fatty acids and carbohydrates in the energy range 0.015-15 MeV up to 40 mean free path. Nucl. Instrum. Methods Phys. Res. B 260:7-19.

29. Kurudirek M, Onaran T. 2015.Calculation of effective atomic number and electron density of essential biomolecules for electron, proton, alpha particle and multi energetic photon interactions. Radiat. Phys. Chem 112:125-138.

30. Van der Schans GP. 1978. Gamma-ray induced double-strand breaks in DN resulting from randomlyinflicted single-strand breaks: temporal local denaturation, a new radiation phenomenon. Int J Radiat Biol Relat Stud Phys Chem Med 33:105-120.

31. Sabbaghi A, Miri SM, Keshavarz M, Zargar M, Ghaemi A. Inactivation methods for whole influenza vaccine production. Rev Med Virol. (2019) 29:e2074.

32. Motamedi-Sedeh F, Soleimanjahi H, Jalilian AR, Mahravani H, Shafaee K, Sotoodeh M, et al. Development of protective immunity against inactivated Iranian isolate of foot-and-mouth disease virus type 0/IRN/2007 using gamma ray-irradiated vaccine on BALB/c mice and Guinea pigs. Intervirology. (2015) 58:190-6.

33. Shahrudin S, Chen C, David SC, Singleton EV, Davies J, Kirkwood CD, et al. Gamma-irradiated rotavirus: a possible whole virus inactivated vaccine. PLoS ONE. (2018) 13:e0198182 
34. Cavanagh, D. Severe Acute Respiratory Syndrome Vaccine Development: Experiences of Vaccination Against Avian Infectious Bronchitis Coronavirus. Avian Pathol.2003, 32, 567-82.

35. Du, L.; He, Y.; Zhou, Y.; Liu, S.; Zheng, B. J.; Jiang, S. The Spike Protein of SARS-CoV-a Target for Vaccine and Therapeutic Development. Nat. Rev. Microbiol.2009, 7, 226-36.

36. Jiang, S.; He, Y.; Liu, S. SARS Vaccine Development. Emerg. Infect. Dis.2005, 11, 1016-1020.

37. Taylor, D. R. Obstacles and Advances in SARS Vaccine Development. Vaccine2006, 24, 863-871.

38. Roper, R. L.; Rehm, K. E. SARS Vaccines: Where Are We? Expert. Rev. Vaccines.2009, 8, 887-898.

39. Harima Y, Kurosawa N, Sakamoto Y. 2014. Parameter search of geometric- progression formula for gamma-ray isotropic point source buildup factors up to depths of $100 \mathrm{mfp}$, including contribution of secondary radiations. J. Nucl. Sci. Tech 4:548-552.

40. Yoshida Y. 2006. Development of Fitting Methods Using Geometric Progression Formulae of Gamma-ray Buildup Factors. J. Nucl. Sci. Tech 43:1446-1457.

41. Chilton AB, Shultis JK, Faw R. 1984. Principle of Radiation Shielding, $1^{\text {st }}$ ed. New Jersey: PrenticHalle, Englewood Cliffs.

42. Bhandal GS, Singh K. 1997. Effective atomic number studies in different biological samples for partial and total photon interactions in the energy range $10^{-3}$ to $10^{5} \mathrm{MeV}$. Int. J. Appl. Radiat. Isot 44:505-510.

43. Globus T, Woolard D, Crowe TW, Khromova T, Gelmont B, et al. (2006) Terahertz fourier transform characterization of biological materials in a liquid phase. J Phys D: Appl Phys 39: 3405-3413.

44. Duong TH, Zakrzewska K (1997) Calculation and analysis of low frequency normal modes for DNA. J Comput Chem 18: 796-811.

45. Micke O, Seegenschmiedt MH, German Working Group on Radiotherapy in G. Consensus guidelines for radiation therapy of benign diseases: a multicenter approach in Germany. Int J Radiat Oncol Biol Phys 2002; 52:496-513.

46. George D. Wilson, Minesh P. Mehta, James S. Welsh, Arnab Chakravarti, C. Leland Rogers and James Fontanesi, Investigating Low-Dose Thoracic Radiation as a Treatment for COVID-19 Patients to Prevent Respiratory Failure, RADIATION RESEARCH 194, 1-8 (2020).

47. Friederike Feldmann, W. Lesley Shupert, Elaine Haddock, Barri Twardoski, and Heinz Feldmann, Gamma Irradiation as an Effective Method for Inactivation of Emerging Viral Pathogens, Am. J. Trop. Med. Hyg., 100(5), 2019, pp. 1275-1277.

\section{Tables}

Table 1: Some modifications of the RSFIEDLLFNKV motif in SARS-CoV-2 of mammalian hosts along with their GeneBank code

Table 2 Amino acids structure and molecular formula and compositions of the investigated S2 subsequence of the SARS-CoV-2 Spike protein in weight fraction. 


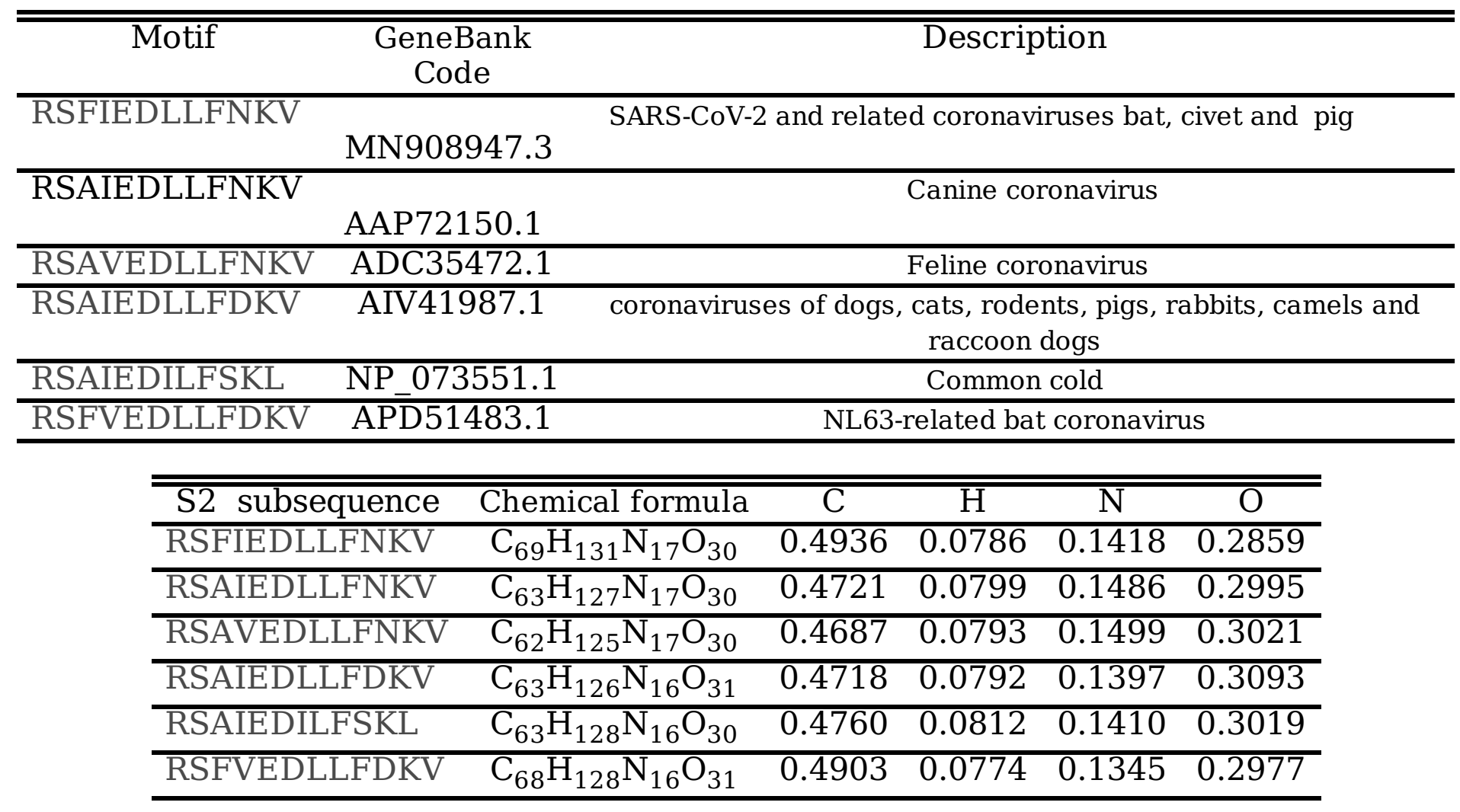

\section{Figures}



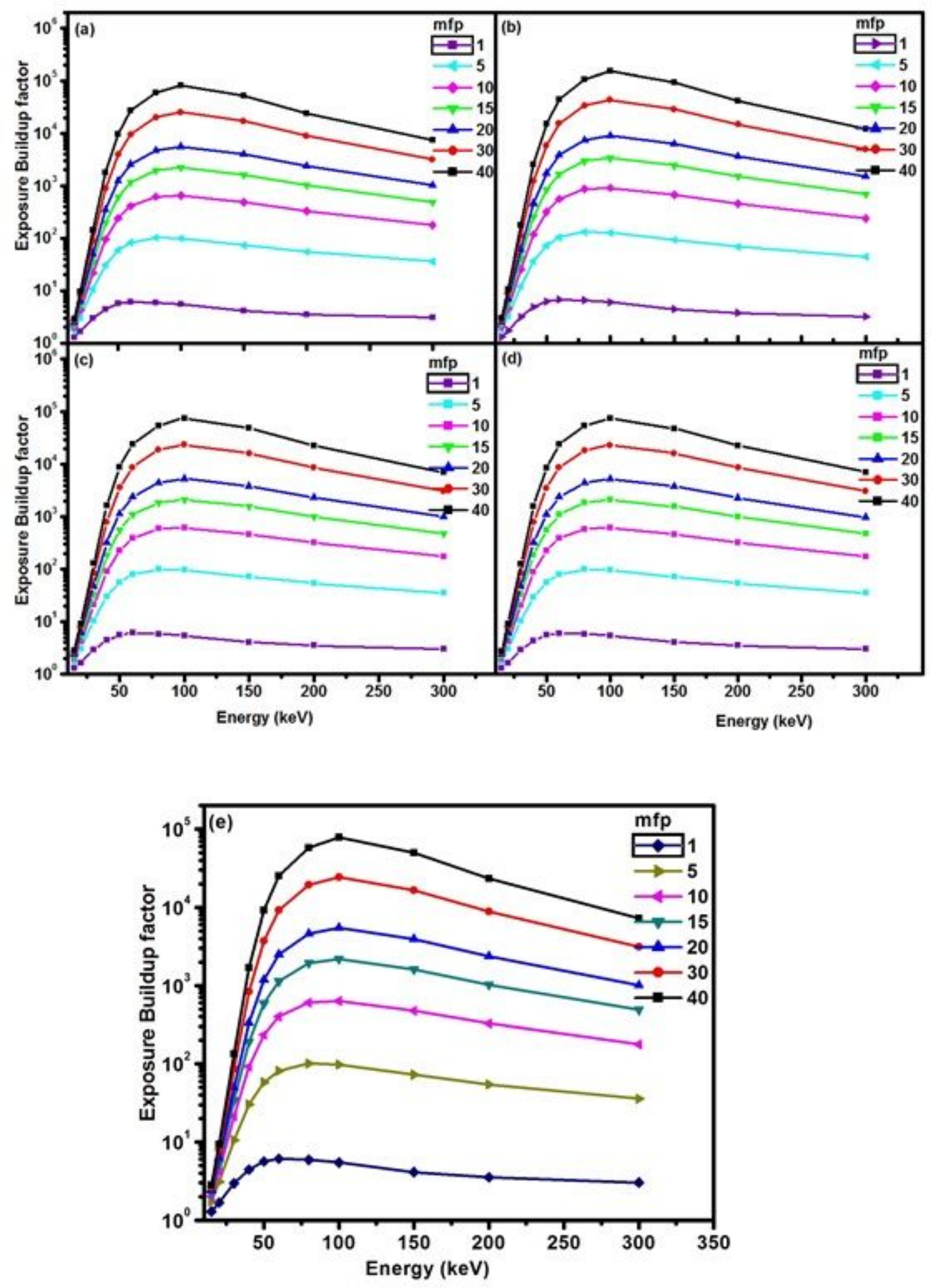

\section{Figure 1}

Variation of energy exposure buildup factors with photon energy for S2 subsequence of SARS-COV-2 Spike protein for the various mutations used in this study with photon energy at selected values of mfp as indicated in the figure. The resonance peak depends on the type of mutation of SARS-COV-2 Spike protein. 

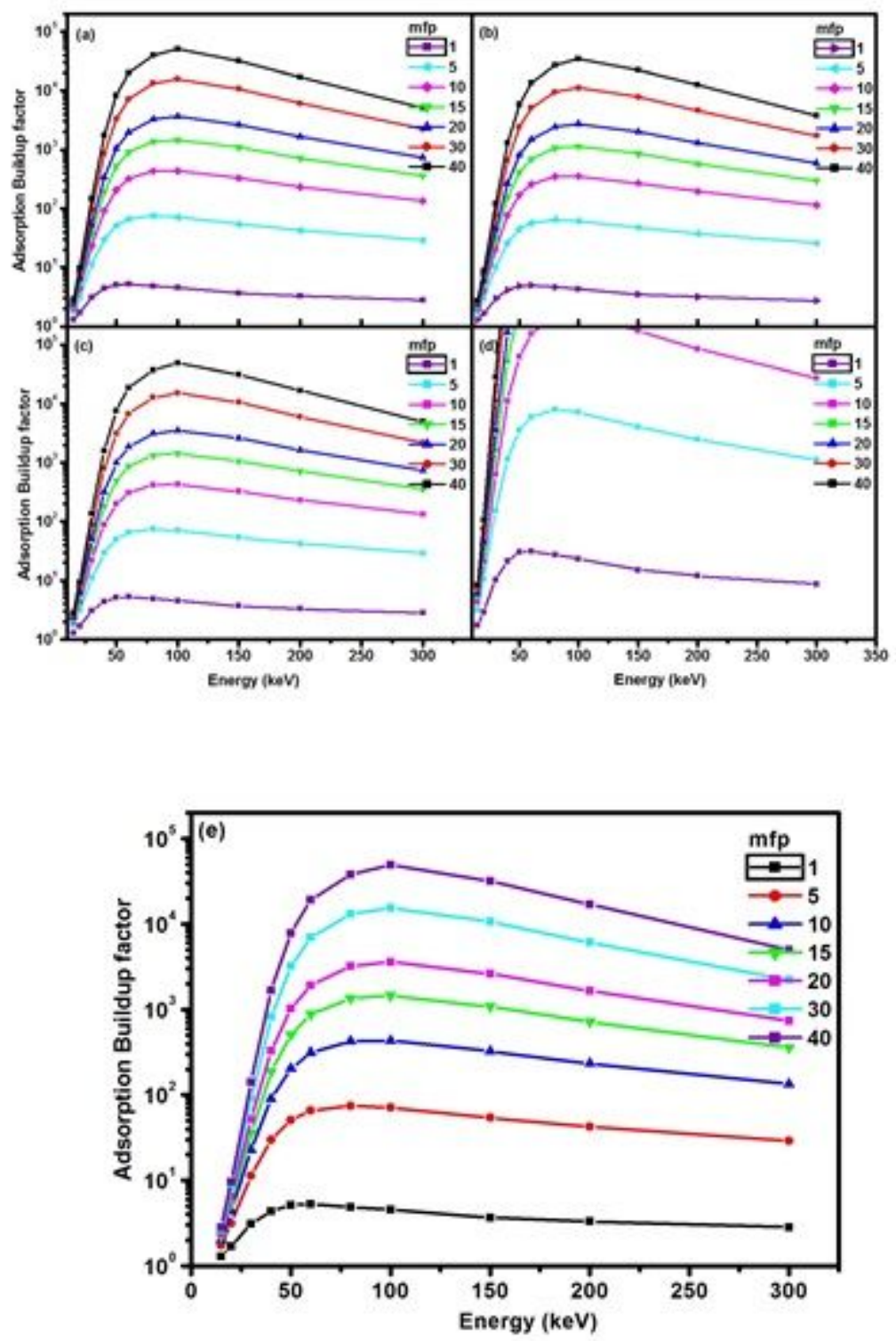

Figure 2

Variation of energy absorption buildup factors for S2 subsequence of SARS-COV-2 Spike protein for the various mutations used in this study with photon energy at different values of $\mathrm{mfp}$ as indicated in the figure.

\section{Supplementary Files}

This is a list of supplementary files associated with this preprint. Click to download.

- Schematic1.jpg

- supplemetalinformation.xls 